\title{
Integrating Imaging Data into Predictive Biomathematical and Biophysical Models of Cancer
}

\author{
Thomas E. Yankeelov ${ }^{1,2,3,4,5,6}$ \\ ${ }^{1}$ Institute of Imaging Science, Vanderbilt University, AA-1105 Medical Center North, 116121 st Avenue South, Nashville, \\ TN 37232-2310, USA \\ ${ }^{2}$ Department of Radiology and Radiological Sciences, Vanderbilt University, AA-1105 Medical Center North, 116121 st Avenue South, \\ Nashville, TN 37232-2310, USA \\ ${ }^{3}$ Department of Biomedical Engineering, Vanderbilt University, AA-1105 Medical Center North, 116121 st Avenue South, \\ Nashville, TN 37232-2310, USA \\ ${ }^{4}$ Department of Physics, Vanderbilt University, AA-1105 Medical Center North, 1161 21st Avenue South, Nashville, \\ TN 37232-2310, USA \\ ${ }^{5}$ Department of Cancer Biology, Vanderbilt University, AA-1105 Medical Center North, 116121 st Avenue South, Nashville, \\ TN 37232-2310, USA \\ ${ }^{6}$ Vanderbilt Ingram Cancer Center, Vanderbilt University, 1161 21st Avenue South, Nashville, TN 37232-2310, USA
}

Correspondence should be addressed to Thomas E. Yankeelov, thomas.yankeelov@vanderbilt.edu

Received 1 November 2012; Accepted 21 November 2012

Academic Editors: J. Chow, S.-C. Ngan, and J. Suehnel

Copyright (C) 2012 Thomas E. Yankeelov. This is an open access article distributed under the Creative Commons Attribution License, which permits unrestricted use, distribution, and reproduction in any medium, provided the original work is properly cited.

\begin{abstract}
While there is a mature literature on biomathematical and biophysical modeling in cancer, many of the existing approaches are not of clinical utility, as they require input data that are extremely difficult to obtain in an intact organism, and/or require a large number of assumptions on the free parameters included in the models. Thus, there has only been very limited application of such models to solve problems of clinical import. More recently, however, there has been increased activity at the interface of quantitative, noninvasive imaging data, and tumor mathematical modeling. In addition to reporting on bulk tumor morphology and volume, emerging imaging techniques can quantitatively report on for example tumor vascularity, glucose metabolism, cell density and proliferation, and hypoxia. In this paper, we first motivate the problem of predicting therapy response by highlighting some (acknowledged) shortcomings in existing methods. We then provide introductions to a number of representative quantitative imaging methods and describe how they are currently (and potentially can be) used to initialize and constrain patient specific mathematical and biophysical models of tumor growth and treatment response, thereby increasing the clinical utility of such approaches. We conclude by highlighting some of the exciting research directions when one integrates quantitative imaging and tumor modeling.
\end{abstract}

\section{Introduction}

The ability to identify-early in the course of therapy-patients that are not responding to a given therapeutic regimen is extraordinarily important. In addition to limiting patients' exposure to the toxicities associated with unsuccessful therapies, it would allow patients the opportunity to switch to a potentially more efficacious approach. Unfortunately, existing methods of determining early response are inadequate. In particular, the current standard-of-care imaging assessment of tumor response to treatment is based on the Response Evaluation Criteria in Solid Tumors (RECIST, [1]). RECIST offers a simplified, practical method for extracting the basic morphological features of imaging data by assessing the overall tumor burden at baseline and comparing that measurement to subsequent measurements obtained during the course of therapy. We now present the salient features of RECIST in order to identify weaknesses that the integration of imaging and mathematical modeling can potentially address. 
High-resolution images (typically computed tomography (CT) or magnetic resonance imaging (MRI)) are acquired at baseline before treatment as commenced. In these image sets, "target lesions" are determined, and the sum of their longest dimensions is recorded. Additional scans are then acquired during or after therapy and similarly analyzed. The change in the sum of the longest diameters from baseline to the follow-up studies are then calculated and then used to divide treatment response into one of the following four categories [1].

(1) Complete response (CR) — disappearance of all target lesions.

(2) Partial response (PR) — at least a 30\% decrease in the sum of the longest diameters of the target lesions, taking as the reference the baseline sum longest diameter.

(3) Progressive disease (PD) — at least a 20\% increase in the sum of the longest diameters of the target lesions, taking as the reference the smallest sum longest diameter recorded since the baseline measurements or the appearance of one or more new lesions.

(4) Stable Disease (SD)—neither sufficient shrinkage to qualify for partial response nor sufficient increase to qualify for progressive disease.

While almost all clinical trials of solid tumors employ the RECIST criteria, it is well recognized that this approach needs to be significantly improved for a number of reasons. For example, the metric for positive response is based on one-dimensional changes (sum of longest diameters of target lesions) which can be grossly misleading in trying to describe a complex object that is almost certainly changing in all three spatial dimensions. Furthermore, this metric is based on anatomical and morphological changes which are (temporally) downstream manifestations of underlying physiological, cellular, or molecular changes; that is, RECIST based evaluations generally do not indicate whether a tumor is responding until the patient has received several treatment cycles. This limitation is particularly problematic in the era of targeted therapies; in fact, it is a matter for debate as to whether the RECIST criteria are even relevant in assessing the activities of non-cytotoxic anti-cancer drugs if changes in morphology may not be the most appropriate method to assess response. What is needed are methods to characterize those underlying physiological, cellular, and molecular changes as they are highly likely to offer earlier and more specific response to treatment indices than changes in longest dimensions. One approach to this shortcoming of RECIST is to begin to incorporate some of the more quantitative and specific noninvasive imaging methods into clinical trials and practice; indeed, there is much current effort at accomplishing this goal (see, e.g., [2-4]). Some progress has been made on this front. For example, in the most recent version of RECIST (RECIST 1.1, [5]), fluorodeoxyglucose positron emission tomography (PET) has now been included in the guidelines, though only in the limited role of assessing progressive disease. This represents the first time a nonmorphology-based imaging measurement has been incorporated into a consensus response criteria. It is anticipated that, in coming years, there will be more physiological, cellular, and molecular imaging measurements adopted into response criteria to compliment the morphology-based assessments.

Independent of the recent developments in the quantitative imaging of cancer, a mature literature has developed in the biomathematical and biophysical modeling of tumor growth (for reviews of this field see, e.g., $[6,7]$ ). The complexity and range of applicability of these models run the gamut from exponential growth of an avascular tumor [8] to elaborate systems of partial differential equations describing cell motility, tumor invasion, and angiogenesis. While these studies have provided many interesting and exciting insights into fundamental cancer biology, they are, however, fundamentally limited in their ability to be translated to clinical application. This is because the majority of these models rely on measurements of processes that occur at the cellular and/or molecular level (growth factor gradients, gene expression levels, etc.) and are therefore extremely difficult to measure in an intact living system. Thus, the fundamental limitation of such approaches is that they are driven by parameters that are accessible only through invasive methods (surgery, biopsy, or animal sacrifice). This leads to the necessity to make multiple assumptions about in vivo parameter values based on, for example, idealized in vitro or ex vivo studies, thus such approaches do not generally lend themselves to patient specific modeling. However, there exist several emerging biomedical imaging methods that can provide quantitative information noninvasively, in $3 \mathrm{D}$, and at multiple time points, thereby providing the opportunity for integrating imaging data into mathematical models to predict the growth and response of tumors to therapy. More specifically, measurements can be made at the time of diagnosis and early in the course of treatment, and these data can be used to initialize and constrain models that predict treatment outcomes. In this way, imaging allows models to be initialized and constrained with patient specific data; furthermore, only by parameterizing models by data that are specific to the individual, can such an approach find clinical utility. If realized, this would mark a significant improvement over, for example, RECIST.

In this paper we aim to show how biomedical imaging data can be used to initialize and constrain patient specific mathematical models of tumor growth to predict tumor status at later time points. To accomplish this task, we first provide brief introductions of a selection of emerging, quantitative, and in vivo imaging measurements which are appropriate for incorporation into predictive biomathematical and biophysical models of tumor growth. We then follow that with a section reviewing the literature where imaging techniques have been successfully incorporated into tumor modeling. The final section includes a discussion of future opportunities at the interface of imaging and modeling and describes three key areas (two theoretical and one experimental) that should be primary goals for investigators working in this exciting and emerging field. 


\section{Data Available from Biomedical Imaging}

We limit our discussion of quantitative imaging metrics to the (currently) most widely available MRI and PET measures. Additionally, these imaging measures are those that have already shown encouraging data in their ability to predict treatment response and are, therefore, natural candidates to integrate into biomathematical and biophysical models of tumor growth and treatment response.

\subsection{Magnetic Resonance Imaging}

2.1.1. Dynamic Contrast Enhanced MRI (DCE-MRI). DCEMRI involves the serial acquisition of MR images of a tissue of interest (e.g., a tumor) before and after an intravenous injection of paramagnetic contrast agent [9]. As the contrast agent enters into the tissue under investigation, the $T_{1}$ and $T_{2}$ values of tissue water decrease to an extent that is determined by the concentration of the contrast; studies that exploit changes in $T_{1}$ are termed DCE-MRI, while those relying on $T_{2}$ changes are termed dynamic susceptibility contrast MRI (DSC-MRI; [10]). By considering a set of images acquired before and after a contrast infusion, a region of interest (ROI) or individual voxels will display a characteristic signal intensity time course, which can be related to the concentration of contrast agent. Fitting the DCE-MRI data to an appropriate pharmacokinetic model allows for extraction of physiological parameters related to, for example, tissue perfusion and microvascular vessel wall permeability (denoted by the parameter $K^{\text {trans }}$ ), extracellular volume fraction (denoted by $v_{e}$ ), and the plasma volume fraction (denoted by $v_{p}$ ). It has been shown that these parameters can change significantly during the course of therapy and be used to predict patients who will have a positive response from those that do not; see, for example, $[4,11-14]$. For a detailed review of DCE-MRI and its applications in cancer, the interested reader is referred to [9].

2.1.2. Diffusion Weighted MRI (DW-MRI). The microscopic thermally-induced behavior of molecules moving in a random pattern is referred to as self-diffusion or Brownian motion [15]. The rate of diffusion in tissues is lower than in free solution and is described by an apparent diffusion coefficient (ADC), which largely depends on the number, permeability and separation of barriers that a diffusing water molecule encounters. MRI methods have been developed to map the ADC, and the variations in ADC have been shown to correlate inversely with tissue cellularity [16]. In regions of high cellularity (e.g., a tumor) there is an increase in cellular membranes thereby providing an increase in the number of barriers encountered by a diffusing water molecule; this results in a decreased ADC. Conversely, when effective cytotoxic therapy reduces the overall cellularity and thereby decreases the number of barriers a diffusing water molecule encounters, there is an increase in the ADC. It has been shown that exposure of tumors to chemotherapy and/or radiotherapy consistently leads to measurable increases in water diffusion in cases of favorable treatment response
[17-19]. For a detailed review of DW-MRI and its applications in cancer, the interested reader is referred to [20].

2.1.3. Diffusion Tensor Imaging (DTI). Diffusion tensor imaging (DTI) is a method to noninvasively characterize the structural connectivity between brain cortical regions. DTI provides, for each voxel, a tensor matrix that describes the constraints on local Brownian motion of water molecules. Originally proposed to assess tissue properties such as diffusion anisotropy [21], DTI has recently evolved to become the primary modality for mapping neuronal fiber tracts in vivo, a technique referred to as DTI tractography. DTI tractography draws upon the principle that the dominant direction of water diffusion coincides with the local tangential direction of fibrous tissue, and integration of the principal diffusion directions permits entire fiber tracts to be delineated [22]. Based on these approaches, a number of streamline-like fiber tracking algorithms have been developed to generate the pathways of fiber connections [22-25]. More recently, a number of investigators have examined both how these fiber tracks affect tumor growth and how the growing tumor affects the fiber tracks [26-28]. For a detailed review of DTI and its applications in cancer, the interested reader is referred to [29].

\subsection{Positron Emission Tomography}

2.2.1. Fluorodeoxyglucose PET (FDG-PET). Fluorodeoxyglucose is a glucose analogue that accumulates in areas of increased glycolytic activity which is a near universal property of cancer. It is well known that the activity of cell surface glucose transporters GLUT-1 and GLUT-3 and the intracellular enzyme hexokinase are upregulated in malignant cancer cells. The transporters function is to transport FDG into tumor cells where it is phosphorylated by hexokinase and trapped (and hence accumulated) in malignant cells. FDGPET has been used extensively in both the preclinical and clinical settings to study treatment response [30,31]. (It is the far and away the most commonly used PET tracer in clinical oncology.) In particular, reductions in the delivery and retention of PET have been shown to correlate to and, in some cases, predict therapeutic response [31-34]. For a detailed review of FDG-PET and its applications in cancer, the interested reader is referred to [35].

2.2.2. Fluorodeoxythymidine PET (PET-FLT). Fluorodeoxythymidine (FLT) was developed as a surrogate marker of cellular proliferation [34]. Once transported to the tumor intracellular space FLT is phosphorylated by thymidine kinase-1 (TK1), which is known to have a large increase in activity during DNA synthase phase of the cell cycle (S phase). Since FLT monophosphate is not incorporated into DNA and is impermeable to the cell membrane, it is metabolically trapped intracellularly. Thus, rapidly proliferating (tumor) cells can present an increased retention of FLT when compared to background (healthy) tissue. As with FDG, there has been much recent interest in FLT in both preclinical and clinical studies of treatment response [34, 3639]. The general working hypothesis for FLT-PET studies 
is that treatments designed to reduce cellular proliferation will show decreased FLT uptake, and this will be evident in responders, whereas nonresponders will not display a decrease in FLT accumulation. Indeed, many recent studies have displayed just such a relationship. For a detailed review of FLT-PET and its applications in cancer, the interested reader is referred to [40].

2.2.3. Hypoxia Imaging via PET. It is well known that hypoxia in malignant tumors can affect the outcome of anticancer treatments, as malignant tumors are more resistant to chemotherapy and irradiative therapy because of their lack of oxygen, which is a potent radiosensitizer [41]. Thus, there has been much effort in developing imaging methods for characterizing tissue oxygen status [42]; in particular, there have been several approaches employing derivatives of misonidazole. Upon entering a cell misonidazole is reduced and retained in hypoxic cells but freely exits the cell under normoxic conditions. Using ${ }^{14} \mathrm{C}$-labeled misonidazole, Chapman was the first to demonstrate the potential of radiolabeled nitroimidazoles for the detection of hypoxia [43]. The clinical detection of hypoxia became available with the introduction of ${ }^{18}$ F-Fluoromisonidazole [44]. Several studies have since demonstrated the clinical prognostic value of FMISO [45, 46]. FMISO is also being used to assess hypoxia and oxygen-enhancing therapeutics in experimental cancer models $[47,48]$. For a detailed review of FMISO-PET and its applications in cancer, the interested reader is referred to [49].

Another common PET-based method for studying at hypoxia is based on copper(II)-diacetyl-bis $\left(N^{4}\right.$-methylthiosemicarbazone), Cu-ATSM, which can be labeled with a positron emitting isotope of copper $\left({ }^{\mathrm{x}} \mathrm{Cu}\right.$, where $\mathrm{x}=60$, 61,62 , or 64) [50]. Owing to its high membrane permeability and low redox potential, ${ }^{x} \mathrm{Cu}-\mathrm{ATSM}$ tracers can access the intracellular space and are stable there under normoxia. However, when entering hypoxic tissue the ${ }^{x} \mathrm{Cu}(\mathrm{II})$ in ${ }^{\mathrm{x}} \mathrm{Cu}-\mathrm{ATSM}$ is reduced to ${ }^{\mathrm{x}} \mathrm{Cu}(\mathrm{I})$ and is released from the ATSM chelate, thereby becoming trapped intracellularly and, therefore, ${ }^{\mathrm{x}} \mathrm{Cu}$-ATSM is preferentially accumulated in hypoxic tissues $[51,52]$. These agents have been used to study, for example, the ability to predict treatment response $[53,54]$ and determine prognosis $[55,56]$. For a detailed review of ${ }^{x} \mathrm{Cu}$-ATSM-PET and its applications in cancer, the interested reader is referred to [57].

2.3. Multimodal Imaging Data. As should be apparent from the above discussions, many of the imaging modalities are complimentary in nature, and this realization has spurred the development of multimodal assessments of cancer. For example, combining the measurements available from DCE-MRI and FMISO-PET can provide insight into the relationship between vascular status and tissue oxygenation. Another obvious paring is DW-MRI and FLT-PET to explore the (temporal and spatial) relationships between cell proliferation and cellularity. To date the overwhelming majority of multimodal studies have been correlative in nature and there have been only minimal efforts at integrating such data into an appropriate biomathematical or biophysical model.
(We review such investigations below in Section 3.2) However, as multimodality imaging of cancer continues to develop [58-60], and clinical PET-MRI scanners come online [61-63], the ability to acquire quantitative, multimodality imaging data becomes increasingly accessible, and consequently, the ability to initialize and constrain mathematical models of tumor growth becomes increasingly realistic. This is an area for which there is great opportunity for investigations of real clinical impact.

\section{Current Efforts at Integrating Imaging and Mathematical Models}

We divide our review of the literature on integrating modeling and imaging into subsections in which a single imaging technique is employed, and a section where multiple imaging techniques are employed. We note that the order of the manuscripts reviewed below was determined by their publication date.

3.1. Modeling Studies Employing One Imaging Technique. As it is well known that glioma cells preferentially migrate along white matter fibers of the brain, it is natural to incorporate the structural a priori information on brain architecture provided by diffusion tensor imaging to guide how tumor cells will move from their source point. This is the approach taken by Jbabdi et al. [64]. The authors began with a reaction-diffusion model of how tumor cells proliferate and migrate as follows:

$$
\frac{\partial c}{\partial t}=D \nabla^{2} c(x, t)+\rho c(x, t),
$$

where $c(x, t)$ is the concentration of tumor cells at position $x$ and time $t, D$ is the diffusion coefficient associated with random tumor cell motion, and $\rho$ is the proliferation rat of the tumor cells. The first term on the right hand side of (1) is the diffusion component, while the "reaction" portion is characterized by the exponential growth term (the second term on the right hand side). As it is written, (1) does not account for the heterogeneous structure of the brain, and one way to incorporate such knowledge is to make $D$ an explicit function of space dependent upon information provided by the DTI data. In this case, we have

$$
\frac{\partial c}{\partial t}=\nabla \cdot(\mathbf{D}(x) \nabla c(x, t))+\rho c(x, t)
$$

where $\mathbf{D}(x)$ is the diffusion tensor that describes anisotropic tumor cell diffusion and therefore takes into account both location and direction as reported by the DTI data. By combining an average value for $\rho$ (obtained from previous investigations $[65,66])$ with appropriate initial (i.e., $c(x, 0)=$ $\left.c_{o}(x)\right)$ and boundary $(\mathbf{D}(x) \nabla c(x, t) t) \cdot \mathbf{n}=0$ for all $x$ on the sulcal and ventricular boundary, where $\mathbf{n}$ is the surface normal) conditions, the model can then be run forward to predict tumor shape and distribution which can then be compared to experimental results. In their initial effort, the authors used DTI data from a healthy volunteer, as it was not available on the patient data sets. The authors 
stated that their goal was to simulate tumor growth in a healthy brain to best fit the tumor size and shape that was experimentally observed in patients with brain tumors lowgrade tumors. By "seeding" a tumor in the healthy brain data set and "growing" it forward in time, they were able to compare to their predicted tumors to those observed in real patients. The results indicated that when the tumors were allowed to grown anisotropically, as guided by the DTI data, the spatial extension and distribution of the tumor more closely resembled what was observed in the patient, then when the tumor cells were allowed to migrate isotropically without guidance from the DTI data. This preliminary study showed the importance of incorporating structural information available from imaging data in order to account for more realistic tumor cell growth.

A similar approach to linking tumor growth and restricted movement was investigated by Clatz et al., who simulated the growth of glioblastoma multiforma (GBM) by incorporating the mechanical restrictions (based on classical continuum mechanics) presented by certain brain structures [67]. Their overall goal was to build a patient-specific simulator of GBM which includes brain deformation induced by the invading tumor. To conceptualize this, the tumor is broken down into two components: (1) the expansive, noninfiltrative component that produces the increase in tumor volume and is responsible for the mass effect and (2) the component associated with tumor diffusion that is responsible for the infiltrative component. The components are coupled in the sense that the mass effect is directly related to tumor cell density, but the tumor cell density is not influenced by the mass effect. To implement this approach, the authors first segment the images into an expansive component and a diffusion component, and the images are then registered to a brain (anatomical) atlas. Then a finiteelement mesh is built based on the segmentations, and tissue properties are assigned to each mesh point including the tumor cell density based on the two segmented components. Based on this initialization, the reaction diffusion equation is run forward to generate a virtual GBM whose results are then compared to the patient data; the simulated tumor isodensity contours and brain deformation are compared to what is actually measured on MR images by registering the predicted tumor to that acquired approximately six months after the initial measurements. The algorithm was applied to two patients, and the predicted tumor sizes and shapes showed good qualitative agreement (no quantitative comparison was performed) to what was actually measured on anatomical $T_{2}$-weighted MR data. These initial results were very encouraging and, importantly, showed the feasibility of using limited clinical imaging data to initialize a patient specific model of tumor growth. The authors concluded by noting that this success justified its further evaluation which then did in a follow-up paper.

Bondiau et al. [68] combined the main features of the previous two paragraphs-DTI measurements of white matter tracks and mechanically restricted tumor growthto model GBM in an excellent paper in 2008. The DTI data is used to estimate the preferred direction of migration for the diffusion component of the reaction diffusion (2) which is then coupled to a mechanical component. The authors again used classical linear elasticity theory to describe how the brain reacts to the tumor. In particular, they assume a linear relation between stress and strain as follows:

$$
\sigma=k \varepsilon,
$$

where $\sigma$ is the stress tensor, $k$ is the elasticity of the brain, and $\varepsilon$ is the linearized Lagrange strain tensor. Since the deformation is assumed to be very slow the authors use the static equilibrium equation which states that the stress induced by tumor growth is balanced by the external force, $F_{\text {ext }}$, due to tissue deformation:

$$
\nabla \cdot \sigma+F_{\mathrm{ext}}=0 .
$$

The model parameters can then be tuned to match that of an MRI data obtained from a patient; that is, the different brain structures have different mechanical properties, and these are assigned in a straightforward fashion by image segmentation and literature values [69]. These assignments, together with the model can then be used to simulate tumor status at a future time point. The simulation is then compared to what is actually observed at that future time point. In this contribution, the authors were able to perform a set of quantitative comparisons including the average and odd ratio of the differences between the simulated and observed images, as well as displacement of reference points. The authors were able to show good correlation between the simulated and measured tumor masses; in particular, they stated that the inclusion of DTI data to "guide" tumor migration improves the "realism" of the diffusion component of (2). More recently, the authors have extended their approach to compare predicted growth patterns with irradiation margins in which they showed that microscopic invasion occurs past the irradiation margins and that some healthy tissue was within the radiation margins [68]. (We return to this important topic below.) Clearly, this is an exciting direction of investigation.

Also using MRI data to drive the reaction-diffusion equation in GBM patients, Wang et al. employed serial pretreatment MRI data to generate patient specific rates of net glioma cell proliferation and invasion in 32 patients [70]. The results in this paper were based on previous efforts by this group in which an imaging-based method was developed to compute the parameters $\rho$ and $D$ from serial pre-treatment MRI data [71]. Briefly, this is done by using the serial $T_{1}$ weighted (postcontrast) images in which the volumes of the tumor mass visible on these image sets were computed, and then the radius of a sphere of an equivalent volume is computed. The difference between the measured radii, along with the time interval between subsequent scans, allows for the calculation of the velocity of radial expansion, $v$. This velocity is then substituted into Fisher's approximation [72]:

$$
v=2 \sqrt{D \rho} .
$$

In addition to the $T_{1}$-weighted images, $T_{2}$-weighted images are collected which allows for calculating $D / \rho$ (the socalled, "index of invisibility" [71]. Given this system of 
two equations in two unknowns it is straight forward to compute $D$ and $\rho$. Using this approach, the authors showed that their estimates of proliferation and invasion proved to be significantly related to prognosis; in particular, the ratio $\rho / D$ was a significant predictor of survival $(P=0.01)$. Additionally, the authors compute a new measure, termed the therapeutic response index (TRI), which is the ratio of the actual patient survival time to the predicted survival time achieved without therapeutic intervention. Thus, the TRI provides a quantitative assessment of the efficacy of a particular treatment paradigm. The authors concluded that their approach of developing patient-specific virtual controls for application in both treatment design as well as monitoring the effects of that treatment.

Rockne et al. extended (2) by adding a term describing the linear-quadratic radiobiological model to describe the effects of radiation therapy [73, 74]. This model considers the surviving fraction, $S$, of cells as

$$
S=\exp \left[-\left(\alpha D_{T}+\beta D_{T}\right)\right]
$$

where $D_{T}$ is total dose administered, and $\alpha$ and $\beta$ are constants from experiment. In practice, $D_{T}$ is fractionated over $n$ fractions, and the fractionated dose, $d$, is a function of both space and time, that is, $d \equiv d(x, t)$. Then the biologically effective dose can be shown to be [74]

$$
\mathrm{BED}=n d\left(1+\frac{d}{\alpha / \beta}\right) .
$$

With these assignments, equation (2) is thus updated to

$$
\begin{aligned}
\frac{\partial c}{\partial t}= & \nabla \cdot(\mathbf{D}(x) \nabla c(x, t))+\rho c(x, t) \\
& -R(\alpha, d(x, t), t) c(x, t)
\end{aligned}
$$

where

$$
\begin{aligned}
& R(\alpha, d(x, t)) \\
& \quad=\left\{\begin{array}{cc}
0, & \text { for } t \text { not during therapy } \\
1-S(\alpha, d(x, t)), & \text { for } t \text { during therapy }
\end{array}\right\} .
\end{aligned}
$$

Using this model, the investigators could simulate how a tumor treated with radiation therapy would compare to an untreated tumor, as well as estimate a "typical response window." The group then moved to apply this methodology to patient data. Using pretreatment MRIs to estimate patient specific $\rho$ and $D$ parameters as described above, Rockne et al. made predictions about what the response to radiation would be and correlated these results to clinical data [75]. In particular, they showed the proliferation rate was highly correlated to the radiation response parameter $(r=$ $0.89 ; P=0.0007)$ which allowed for the construction of a predictive relationship that was then tested in a "leave-oneout" approach. This result let the authors to conclude that their model can generate in silico tumors, with characteristics similar to the in vivo (i.e., patient) tumors and that this framework can then be used to predict individual response.
Our own group has recently contributed a simple approach to integrating imaging and modeling, whereby serial DW-MRI data obtained before and early after the initiation of therapy are used to populate the logistic growth model [76-79] and predict tumor cellularity at the conclusion of therapy. The goal is to use the measurement of the ADC to first convert to estimates of cell number [16], and then use changes in the cell number to convert to a proliferation value. More specifically, we convert measurements of ADC to tumor cell number via (10) as follows:

$$
N(t)=\theta\left(\frac{\mathrm{ADC}_{w}-\mathrm{ADC}(t)}{\mathrm{ADC}_{w}-\mathrm{ADC}_{\text {min }}}\right),
$$

where $\mathrm{ADC}_{w}$ and $\mathrm{ADC}_{\text {min }}$ are the $\mathrm{ADC}$ of free water and the voxel with the minimum $\mathrm{ADC}$, respectively; the voxel with the minimum $\mathrm{ADC}\left(\mathrm{ADC}_{\mathrm{min}}\right)$ contains the maximum number of cells that can fit into that voxel (i.e., the carrying capacity, $\theta$. The voxel with the $\mathrm{ADC}$ of free water $\left(\mathrm{ADC}_{w}\right)$ is taken to have zero tumor cells. Thus, given ADC data it is straightforward to convert to tumor cell number. If this approach is applied at multiple time points, it is possible to computer the proliferation rate, $k$, of the tumor on a region of interest or voxel level by fitting the $N(t)$ data to, say, (11):

$$
N(t)=\frac{\theta N\left(t_{1}\right)}{N\left(t_{1}\right)+\left(\theta-N\left(t_{1}\right)\right) \mathrm{e}^{-k t}} .
$$

(We note that the parameter $k$ is actually a bulk (averaged) measure of both proliferation and death and can therefore take on either positive or negative values.) Our first effort [77] using this simple approach was in a small animal model of cancer in which 13 rats were given 9L gliosarcoma brain tumors; eight of the rats were treated with the chemotherapeutic drug 1,3-bis(2-chloroethyl)-1-nitrosourea, and five rats were untreated controls. All animals underwent DWMRI immediately before, one day and three days after treatment. The ADC data from the first imaging time points were used to estimate $k$ as just described which was then used to predict the number of tumor cells at data three, and this value was correlated with the day five experimental data. On a region of interest basis, the Pearson's correlation coefficients between the predicted and measured tumor cellularity was 0.88 . Thus, this simple approach has some ability to capture the gross (i.e., at the ROI level) cellularity changes.

We also applied this approach to breast cancer data acquired during neoadjuvant chemotherapy. Six patients received DW-MRI before, after one cycle, and after all cycles of neoadjuvant chemotherapy. Again, the proliferation rates were estimated using the ADC data from the first two time points and then used with (10) and (11) to predict cellularity after therapy. When comparing the predicted to the experimentally observed data, the Pearson's correlation coefficient for the ROI analysis yielded $0.95(P=0.004)$, and, encouragingly, after applying a conservative $3 \times 3$ mean filter to the ADC data, the voxel-by-voxel analysis yielded a Pearsons correlation coefficient of $0.70 \pm 0.10$ $(P<0.05)$. More recently, we have had some preliminary success in using this approach to separate responders from nonresponders [80]. 
Another effort that has incorporated serially acquired DW-MRI data into a mathematical model was contributed by Ellingson et al. [81]. As there is an established negative correlation between ADC and cellularity [16], the ADC can be directly substituted into (2) to capture

$$
\frac{d}{d t} \mathrm{ADC}=\nabla \cdot(D \nabla \mathrm{ADC})+\rho \mathrm{ADC},
$$

and, subsequently,

$$
\frac{d}{d t} \mathrm{ADC}=D \nabla^{2} \mathrm{ADC}+\nabla D \cdot \nabla \mathrm{ADC}+\rho \mathrm{ADC} .
$$

Algebraically solving (13) for $\rho$ at time point $n-1$ allows for an estimate of $\rho$; that is,

$$
\begin{aligned}
\rho^{n-1}= & \left(\frac{1}{\mathrm{ADC}^{n-1}}\right) \\
& \times\left(\frac{d}{d t} \mathrm{ADC}^{n-1}-D \nabla^{2} \mathrm{ADC}^{n-1}-\nabla D \cdot \nabla \mathrm{ADC}^{n-1}\right),
\end{aligned}
$$

where $d / d t$ of $\mathrm{ADC}^{n-1}$ defines the rate of change of ADC from the previous time point $n-1$ to the first time point $n-2$. After $\rho$ is fixed, it is substituted back into (12) to yield a PDE that can be solved to estimate $D$. The authors applied this approach to obtain estimates of $\rho, D$, and $v$ in 53 patients. In nine of the patients, magnetic resonance spectroscopy was performed to measured Cho/NAA (i.e., choline to N-acetylaspartate ratio); the Pearson's correlation coefficient between $\rho$ and Cho/NAA was $0.97(P<0.0001)$. Furthermore, using a "hot-spot analysis" (based on abnormal anatomical MRI regions), the authors were able to use $\rho$ and $D$ to statistically separate (World Health Organization, WHO) grade II from grades III and IV. The authors then extended this effort to predict progression free and overall survival (OS) in 26 recurrent glioblastoma patients treated with the antiangiogenic drug bevacizumab [82]. Again, the authors were able to find strong correlations between $\rho$ and progression-free survival $(P<0.0001)$ and overall survival $(P=0.0093)$.

3.2. Modeling Studies Employing Multiple Imaging Techniques. One of the first studies to include multimodality imaging in a mathematical model of tumor growth was put forth by Titz and Jeraj in 2008 [83]. In this study, the authors employed FDG-PET/CT data to investigate the effects of oxygenate status on the response to radiation therapy. The CT data are used to extract the bulk tumor size and shape. The molecular imaging data they incorporated into the modeling was FLT-PET and ${ }^{61} \mathrm{Cu}$-ATSM-PET [84] data of head and neck cancer. This data is incorporated into an extension of the standard linear-quadratic model to describe the effects of radiation therapy. The authors' goal was to explicitly incorporate the effects of oxygen status on the linear-quadratic model so they include an addition "oxygendependent modification factor," or OMF, into the relation.
Thus, the survival probability for cells being irradiated is given by

$$
\begin{aligned}
& P\left(D, p \mathrm{O}_{2}\right) \\
& =\exp \left[-\alpha D \cdot \operatorname{OMF}\left(p \mathrm{O}_{2}\right) \cdot\left(1+\frac{D \cdot \mathrm{OMF}\left(p \mathrm{O}_{2}\right)}{\alpha / \beta}\right)\right],
\end{aligned}
$$

where $\alpha$ and $\beta$ are the linear and quadratic contributions, respectively, of the absorbed dose $D$ to the induced damage. The OMF is then given by the oxygen enhancement ratio (OER) at the current (relative) partial pressure of oxygen, $\mathrm{pO}_{2}$ :

$$
\operatorname{OER}\left(p \mathrm{O}_{2}\right)=\frac{m \cdot p \mathrm{O}_{2}+K}{p \mathrm{O}_{2}+K}
$$

where $m$ is the maximum OER, and $K$ is the $p \mathrm{O}_{2}$ at the midpoint between 1 and $m$. The ${ }^{61} \mathrm{Cu}$-ATSM data is then used to determine the $p \mathrm{O}_{2}$ and the SUV of the FLT-PET data are used to initialize the tumor cell proliferation for each voxel. The main result of the paper is to employ pretreatment FLT-PET and ${ }^{61} \mathrm{Cu}$-ATSM data to initialize their model with the nonuniform $p \mathrm{O}_{2}$ map obtained from the ${ }^{61} \mathrm{Cu}-$ ATSM data, a uniform $p \mathrm{O}_{2}$ of $2.5 \mathrm{mmHg}$, a uniform $p \mathrm{O}_{2}$ of $5.0 \mathrm{mmHg}$, and a uniform $p \mathrm{O}_{2}$ of $9.0 \mathrm{mmHg}$. The simulation showed that the greater the hypoxic fraction is (tissue is generally considered hypoxic if $\mathrm{pO}_{2}$ dips below $2.5 \mathrm{mmHg}$ ) the less efficacious the treatment is. In particular, using the inhomogeneous ${ }^{61} \mathrm{Cu}$-ATSM scan resulted in inhomogeneous tumor response with the least treatment benefit coming in regions of hypoxia. The authors note that this suggests that uniform dose distributions may result in an increased tumor cell survival in hypoxic regions. The authors concluded that their multiscale approach can successfully integrate molecular imaging data into a tumor growth and radiation therapy response tumor simulation.

The first study to merge modeling with clinical MRI and PET data was contributed by Szeto et al. [85] who investigated the link between tumor growth kinetics and hypoxic tumor burden. The growth kinetics were examined using the approach summarized by (2), only this time the proliferation term (second term on the right hand side of (2)) was modeled by logistic growth; that is, there operational equation was

$$
\frac{\partial c}{\partial t}=\nabla \cdot(\mathbf{D}(x) \nabla c(x, t))+\rho c(x, t)\left(1-\frac{c(x, t)}{k}\right),
$$

where $k$ is the carrying capacity of the section of tissue under investigation. The investigators studied the correlation between the relative hypoxic fraction, (1) cell proliferation, and (2) the modeling assessment of "biological aggressiveness." More specifically, the relative hypoxic (RH) fraction was defined as the ratio of the hypoxic volume, as assessed by FMISO-PET, to the tumor volume defined by anatomical $T_{2}$-weighted MRI. Cell proliferation is quantified by $\rho$, while biological aggressiveness is defined as $\rho / D$; both $\rho$ and $D$ are determined as described previously. Performing correlation 
analysis, they were able to show a strong $\left(R^{2}=0.88\right)$ and significant $(P=0.000021)$ relationship between $\rho / D$ and RH. Additionally, a strong $\left(R^{2}=0.74\right)$ and significant $(P=0.00069)$ relationship was also found between $\rho / D$ and the mean FMISO activity of the tumor scaled to the blood FMISO activity. The authors concluded that their data indicates that biological aggressiveness of brain tumors (as quantified by $\rho / D$ ) is linked to hypoxic burden.

Building on these efforts, this group has more recently extended the model described by (17) to include the effects of hypoxia, necrosis, and angiogenesis [86]. This new model divides the population of tumor cells into three subpopulations: normoxic, hypoxic, and dead. Thus, (17) is modified into a system of PDEs with one each for normoxic, hypoxic, dead cells, tumor angiogenic factors, and vasculature. Cells are allowed to convert from one population to the other and that is determined, in part, by vascular and hypoxic status. In this preliminary effort, the authors used this approach combined with two presurgical MRI scans to predict what an FMISO image would look like just prior to surgery. The simulated image is then compared to the experimentally measured one, and it is found that several of the key features of the real image are captured by the simulation.

3.3. Two Points of Interest. Before leaving this section, we stress two points of interest. The first is that the overwhelming majority of the studies linking mathematical modeling and tumor growth or treatment response have occurred in the brain. While the reasons for this are debatable (though the rigid structure of the skull and the presence of many internal fiducial markers facilitates image registration and, therefore, the application of several of the models described above), it is clearly a limitation in the current literature. Moving forward, the mathematical and biophysical modeling communities will need to address the current paucity of application of their techniques at disease sites outside of the brain. The second point worth noting is that the studies described above all have, at their core, the reactiondiffusion equation. As the excellent efforts described above convincingly indicate, this approach has much merit. But integrating the myriad of data available from quantitative imaging will most likely require new modeling approaches.

\section{Future Directions}

There are three key areas (two theoretical and one experimental) that are primary goals for investigators working at the interface of biomedical imaging and tumor modeling: (1) the need to construct models amenable to the incorporation of imaging data, (2) the construction of a framework, informed by clinical and cancer biology, that allows for in silico, individualized clinical, trials, and (3) the design and execution of appropriately designed experiments to test the theoretical developments of areas (1) and (2).

The majority of existing biomathematical and biophysical models of tumor growth and treatment response are not of the form that are readily amenable to incorporating imaging data; more specifically, the models are not of the kind that can readily be populated by data that can be measured in an intact system at multiple time points with reasonable spatial resolution in 3D. Frequently, the models have a wide variety of parameters that must be estimated or assumed based on measurements from disparate sources. Conversely, the utility of imaging data is that it can be acquired on a patient specific basis so that models can be initialized and constrained by an individual patient's characteristics. As the majority of current models are not constructed with this in mind, this is a fundamentalthough artificial-barrier to progress on integrating imaging data in tumor growth models. Thus, going forward, models need to be constructed with the types of measurements that are available from imaging data in mind. In the author's view, this should be one of the primary goals for investigators working in this field.

Once tumor growth models are built that are readily populated by the imaging data described above in Section 3, it becomes immediately possible to perform in silico experiments to predict, given a set of possible treatment regiments, an optimal treatment strategy. For example, in a wellvascularized tumor it would be important to determine the order and timing of (say) an antiangiogenic drug and a cytoxic drug (for an excellent study on this point, the interested reader is referred to [87]). With an appropriate model in hand, simulations could be run in which the order, dosing, and timing of the antiangiogenic and cytoxic drugs were varied, and this would, presumably, lead to different outcomes (i.e., predictions). Thus, the order, dosing, and timing of the drugs could be varied and explored as parameters to be optimized to yield a prediction of what treatment protocol would be most effective. Of course, such studies would require intimate involvement from experts in cancer biology as well as clinical oncologists with expertise in more standard clinical trials. But if such studies were carried out, they would make concrete predictions that are eminently testable in both the preclinical and clinical settings. In this way, the synthesis of imaging and modeling would truly enable the development of a field of "theoretical oncology."

As imaging data is acquired with minimal interaction with the system under investigation, measurements made early in an experiment can lead to predictions that can then be tested later in the experiment. More specifically, in the context of pre-clinical rodent tumor studies, imaging measurements could be made at baseline (i.e., after an implanted tumor reaches a particular size but before therapy commences), then at a second time point early in the course of treatment. These two data sets could then be used to initialize the model(s) to determine the optimal treatment approach as outlined in the previous two paragraphs. The optimized therapy could then be given to the (say) mouse and the disease progression/regression tracked. In this way the predictions of the imaging based model can be directly compared to experiment to determine their in vivo utility and validity. There is no other currently available approach that enables this. Indeed, it is hard to imagine a methodology such as this that does not involve imaging since it is all based on making spatially resolved, noninvasive measurements; any method that can achieve this is some brand of imaging, 
almost by definition. Furthermore, there is, of course, no reason the same paradigm cannot be applied to human clinical trials. Thus, an appropriate model, initialized by patient specific imaging data, could be used to perform a wide range of in silico clinical trials to determine an optimized, personalized treatment approach for patients. Such a development would hasten the arrival of personalized medicine for oncology patients.

\section{Summary and Conclusion}

After reviewing a representative sampling of the emerging, quantitative in vivo imaging data that is readily available, we proceeded to show such data can potentially be used to initialize and constrain biomathematical and biophysical models of tumor growth and treatment response. As quantitative, imaging data can be acquired noninvasively, in $3 \mathrm{D}$, and at multiple time points, it is a natural and powerful method for acquiring data that can directly populate appropriate models. Furthermore, precisely since the data are acquired noninvasively, the model predictions can then be directly compared to what actually happens in the subject from which the data were taken. Thus, this approach lends itself to make patient specific predictions that can be experimentally tested. It is the author's hope that this paper will motivate others to work at the exciting and clinically relevant, interface of quantitative cancer imaging and modeling of tumor growth and treatment response. If such a line of investigation proves successful, then it has the ability to fundamentally shift existing paradigms of therapy selection and monitoring in cancer.

\section{Acknowledgments}

The author would like to thank the National Cancer Institute for support through 1U01CA142565, R01 CA138599, 1P50 098131, P30 CA68485, and R25CA092043, R25CA136440 and the Kleberg Foundation for their generous support of our imaging program. Additionally, the author would like to thank Drs. Vito Quaranta, M.D. Mike Miga, Ph.D. Nkiruka Atuegwu, Ph.D., Jared Weis, Ph.D., and Mr. David Hormuth for many informative and engaging discussions on the topic of integrating mathematical modeling and biomedical imaging.

\section{References}

[1] P. Therasse, S. G. Arbuck, E. A. Eisenhauer et al., "New guidelines to evaluate the response to treatment in solid tumors," Journal of the National Cancer Institute, vol. 92, no. 3, pp. 205216, 2000.

[2] L. R. Arlinghaus, X. Li, M. Levy et al., "Current and future trends in magnetic resonance imaging assessments of the response of breast tumors to neoadjuvant chemotherapy," Journal of Oncology, vol. 2010, Article ID 919620, 17 pages, 2010.

[3] R. L. Wahl, H. Jacene, Y. Kasamon, and M. A. Lodge, "From RECIST to PERCIST: evolving considerations for PET response criteria in solid tumors," Journal of Nuclear Medicine, vol. 50, supplement 1, pp. 122S-150S, 2009.
[4] M.-C. Asselin, J. P. B. O’Connor, R. Boellaard, N. A. Thacker, and A. Jackson, "Quantifying heterogeneity in human tumours using MRI and PET," European Journal of Cancer, vol. 48, no. 4, pp. 447-455, 2012.

[5] E. A. Eisenhauer, P. Therasse, J. Bogaerts et al., "New response evaluation criteria in solid tumours: revised RECIST guideline (version 1.1)," European Journal of Cancer, vol. 45, no. 2, pp. 228-247, 2009.

[6] A. R. A. Anderson and V. Quaranta, "Integrative mathematical oncology," Nature Reviews Cancer, vol. 8, no. 3, pp. 227-234, 2008.

[7] K. A. Rejniak and A. R. A. Anderson, "State of the art in computational modelling of cancer," Mathematical Medicine and Biology, vol. 29, no. 1, pp. 1-2, 2012.

[8] A. H. Juffer, U. Marin, O. Niemitalo, and J. Koivukangas, "Computer modeling of brain tumor growth," Mini Reviews in Medicinal Chemistry, vol. 8, no. 14, pp. 1494-1506, 2008.

[9] T. E. Yankeelov, J. C. Gore, and J. C. Dynamic, "Dynamic contrast enhanced magnetic resonance imaging in oncology: theory, data acquisition, analysis, and examples," Current Medical Imaging Reviews, vol. 3, no. 2, pp. 91-107, 2009.

[10] E. S. Paulson and K. M. Schmainda, "Comparison of dynamic susceptibility-weighted contrast-enhanced MR methods: recommendations for measuring relative cerebral blood volume in brain tumors," Radiology, vol. 249, no. 2, pp. 601-613, 2008.

[11] H. Dongfeng, M. Daqing, and J. Erhu, "Dynamic breast magnetic resonance imaging: pretreatment prediction of tumor response to neoadjuvant chemotherapy," Clinical Breast Cancer, vol. 12, no. 2, pp. 94-101, 2012.

[12] J. Guo, W. E. Reddick, J. O. Glass et al., "Dynamic contrastenhanced magnetic resonance imaging as a prognostic factor in predicting event-free and overall survival in pediatric patients with osteosarcoma," Cancer, vol. 118, no. 15, pp. 3776-3785, 2012.

[13] M. E. Loveless, D. Lawson, M. Collins et al., "Comparisons of the efficacy of a Jak1/2 inhibitor (AZD1480) with a VEGF signaling inhibitor (cediranib) and sham treatments in mouse tumors using DCE-MRI, DW-MRI, and histology," Neoplasia, vol. 14, no. 1, pp. 54-64, 2012.

[14] M. A. Zahra, L. T. Tan, A. N. Priest et al., "Semiquantitative and quantitative dynamic contrast-enhanced magnetic resonance imaging measurements predict radiation response in cervix csancer," International Journal of Radiation Oncology Biology Physics, vol. 74, no. 3, pp. 766-773, 2009.

[15] A. Einstein, "Über die von der molekularkinetischen Theorie der Wärme geforderte Bewegung von in ruhenden Flüssigkeiten suspendierten Teilchen," Annalen der Physik, vol. 322, no. 8, pp. 549-560, 1905.

[16] A. W. Anderson, J. Xie, J. Pizzonia, R. A. Bronen, D. D. Spencer, and J. C. Gore, "Effects of cell volume fraction changes on apparent diffusion in human cells," Magnetic Resonance Imaging, vol. 18, no. 6, pp. 689-695, 2000.

[17] S. Bonekamp, C. P. Corona-Villalobos, and I. R. Kamel, "Oncologic applications of diffusion-weighted MRI in the body," Journal of Magnetic Resonance Imaging, vol. 35, no. 2, pp. 257-279, 2012.

[18] C. Fu, D. Bian, F. Liu, X. Feng, W. Du, and X. Wang, "The value of diffusion-weighted magnetic resonance imaging in assessing the response of locally advanced cervical cancer to neoadjuvant chemotherapy," International Journal of Gynecological Cancer, vol. 22, no. 6, pp. 1037-1043, 2012. 
[19] S. Yoshida, F. Koga, S. Kobayashi et al., "Role of diffusionweighted magnetic resonance imaging in predicting sensitivity to chemoradiotherapy in muscle-invasive bladder cancer," International Journal of Radiation Oncology Biology Physics, vol. 83, no. 1, pp. e21-e27, 2012.

[20] D. M. Patterson, A. R. Padhani, and D. J. Collins, "Technology insight: water diffusion MRI: a potential new biomarker of response to cancer therapy," Nature Clinical Practice Oncology, vol. 5, no. 4, pp. 220-233, 2008.

[21] P. J. Basser, J. Mattiello, and D. LeBihan, "MR diffusion tensor spectroscopy and imaging," Biophysical Journal, vol. 66, no. 1, pp. 259-267, 1994.

[22] S. Mori and P. C. van Zijl, "Fiber tracking: principles and strategies: a technical review," NMR in Biomedicine, vol. 15, no. 7-8, pp. 468-480, 2002.

[23] X. Golay, H. Jiang, P. C. van Zijl, and S. Mori, "High-resolution isotropic 3D diffusion tensor imaging of the human brain," Magnetic Resonance in Medicine, vol. 47, no. 5, pp. 837-843, 2002.

[24] G. J. M. Parker, C. A. M. Wheeler-Kingshott, and G. J. Barker, "Estimating distributed anatomical connectivity using fast marching methods and diffusion tensor imaging," IEEE Transactions on Medical Imaging, vol. 21, no. 5, pp. 505-512, 2002.

[25] A. Mishra, Y. Lu, J. Meng, A. W. Anderson, and Z. Ding, "Unified framework for anisotropic interpolation and smoothing of diffusion tensor images," NeuroImage, vol. 31, no. 4, pp. 1525-1535, 2006.

[26] E. R. Gerstner and A. G. Sorensen, "Diffusion and diffusion tensor imaging in brain cancer," Seminars in Radiation Oncology, vol. 21, no. 2, pp. 141-146, 2011.

[27] A. Roniotis, G. C. Manikis, V. Sakkalis, M. E. Zervakis, I. Karatzanis, and K. Marias, "High-grade glioma diffusive modeling using statistical tissue information and diffusion tensors extracted from atlases," IEEE Transactions on Information Technology in Biomedicine, vol. 16, no. 2, Article ID 6041031, pp. 255-263, 2012.

[28] S. Kim, S. Pickup, O. Hsu, and H. Poptani, "Diffusion tensor MRI in rat models of invasive and well-demarcated brain tumors," NMR in Biomedicine, vol. 21, no. 3, pp. 208-216, 2008.

[29] D. Leclercq, C. Delmaire, N. M. de Champfleur, J. Chiras, and S. Lehéricy, "Diffusion tractography: methods, validation and applications in patients with neurosurgical lesions," Neurosurgery Clinics of North America, vol. 22, no. 2, pp. 253268, 2011.

[30] E. L. Rosen, W. B. Eubank, and D. A. Mankoff, "FDG PET, PET/CT, and breast cancer imaging," Radiographics, vol. 27, supplement 1, pp. S215-S229, 2007.

[31] E. Skoura, I. E. Datseris, I. Platis, G. Oikonomopoulos, and K. N. Syrigos, "Role of positron emission tomography in the early prediction of response to chemotherapy in patients with non-small-cell lung cancer," Clinical Lung Cancer, vol. 13, no. 3, pp. 181-187, 2012.

[32] T. Cazaentre, F. Morschhauser, M. Vermandel et al., "Pretherapy ${ }^{18} \mathrm{~F}-\mathrm{FDG}$ PET quantitative parameters help in predicting the response to radioimmunotherapy in non-hodgkin lymphoma," European Journal of Nuclear Medicine and Molecular Imaging, vol. 37, no. 3, pp. 494-504, 2010.

[33] J. Duch, D. Fuster, M. Muñoz et al., " ${ }^{18}$ F-FDG PET/CT for early prediction of response to neoadjuvant chemotherapy in breast cancer," European Journal of Nuclear Medicine and Molecular Imaging, vol. 36, no. 10, pp. 1551-1557, 2009.
[34] F. Lordick, "The role of PET in predicting response to chemotherapy in oesophago-gastric cancer," Acta Gastro-Enterologica Belgica, vol. 74, no. 4, pp. 530-535, 2011.

[35] J. Czernin, "Oncological applications of FDG-PET," in PET: Molecular Imaging and Its Biological Applications, M. Phelps, Ed., pp. 270-321, Springer, New York, NY, USA, 2004.

[36] D. A. Mankoff, A. F. Shields, and K. A. Krohn, "PET imaging of cellular proliferation," Radiologic Clinics of North America, vol. 43, no. 1, pp. 153-167, 2005.

[37] A. Salskov, V. S. Tammisetti, J. Grierson, and H. Vesselle, "FLT: measuring tumor cell proliferation in vivo with positron emission tomography and $3^{\prime}$-deoxy- $3^{\prime}-\left[{ }^{18} \mathrm{~F}\right]$ Fluorothymidine," Seminars in Nuclear Medicine, vol. 37, no. 6, pp. 429-439, 2007.

[38] L. Kenny, R. C. Coombes, D. M. Vigushin, A. Al-Nahhas, S. Shousha, and E. O. Aboagye, "Imaging early changes in proliferation at 1 week post chemotherapy: a pilot study in breast cancer patients with $3^{\prime}$-deoxy- $3^{\prime}-\left[{ }^{18} \mathrm{~F}\right]$ fluorothymidine positron emission tomography," European Journal of Nuclear Medicine and Molecular Imaging, vol. 34, no. 9, pp. 1339-1347, 2007.

[39] H. J. Sohn, Y. J. Yang, J. S. Ryu et al., “[ $\left.{ }^{18} \mathrm{~F}\right]$ fluorothymidine positron emission tomography before and 7 days after gefitinib treatment predicts response in patients with advanced adenocarcinoma of the lung," Clinical Cancer Research, vol. 14, no. 22, pp. 7423-7429, 2008.

[40] D. Soloviev, D. Lewis, D. Honess, and E. Aboagye, " $\left[{ }^{18}\right.$ F]FLT: an imaging biomarker of tumour proliferation for assessment of tumour response to treatment," European Journal of Cancer, vol. 48, no. 4, pp. 416-424, 2012.

[41] J. Overgaard, "Hypoxic radiosensitization: adored and ignored," Journal of Clinical Oncology, vol. 25, no. 26, pp. 40664074, 2007.

[42] M. Sørensen, M. R. Horsman, P. Cumming, O. L. Munk, and S. Keiding, "Effect of intratumoral heterogeneity in oxygenation status on FMISO PET, autoradiography, and electrode $\mathrm{Po}_{2}$ measurements in murine tumors," International Journal of Radiation Oncology Biology Physics, vol. 62, no. 3, pp. 854-861, 2005.

[43] J. D. Chapman, "Hypoxic sensitizers—implications for radiation therapy," The New England Journal of Medicine, vol. 301, no. 26, pp. 1429-1432, 1979.

[44] W. J. Koh, J. S. Rasey, M. L. Evans et al., "Imaging of hypoxia in human tumors with [F-18]Fluoromisonidazole," International Journal of Radiation Oncology Biology Physics, vol. 22, no. 1, pp. 199-212, 1992.

[45] K. Hirata, S. Terasaka, T. Shiga et al., "18 F-fluoromisonidazole positron emission tomography may differentiate glioblastoma multiforme from less malignant gliomas," European Journal of Nuclear Medicine and Molecular Imaging, vol. 39, no. 5, pp. 760-770, 2012.

[46] M. Kikuchi, T. Yamane, S. Shinohara et al., " 18 F-fluoromisonidazole positron emission tomography before treatment is a predictor of radiotherapy outcome and survival prognosis in patients with head and neck squamous cell carcinoma," Annals of Nuclear Medicine, vol. 25, no. 9, pp. 1-9, 2011.

[47] C. Oehler, J. A. O’Donoghue, J. Russell et al., " ${ }^{18}$ F-fluromisonidazole PET imaging as a biomarker for the response to 5,6-dimethylxanthenone-4-acetic acid in colorectal xenograft tumors," Journal of Nuclear Medicine, vol. 52, no. 3, pp. 437444, 2011.

[48] R. Bejot, V. Kersemans, C. Kelly, L. Carroll, R. C. King, and V. Gouverneur, "Pre-clinical evaluation of a 3-nitro-1,2,4triazole analogue of $\left[{ }^{18} \mathrm{~F}\right] \mathrm{FMISO}$ as hypoxia-selective tracer 
for PET," Nuclear Medicine and Biology, vol. 37, no. 5, pp. 565$575,2010$.

[49] S. T. Lee and A. M. Scott, "Hypoxia positron emission tomography imaging With ${ }^{18}$ F-Fluoromisonidazole," Seminars in Nuclear Medicine, vol. 37, no. 6, pp. 451-461, 2007.

[50] Y. Yoshii, M. Yoneda, M. Ikawa et al., "Radiolabeled Cu-ATSM as a novel indicator of overreduced intracellular state due to mitochondrial dysfunction: studies with mitochondrial DNAless $\rho^{0}$ cells and cybrids carrying MELAS mitochondrial DNA mutation," Nuclear Medicine and Biology, vol. 39, no. 2, pp. 177-185, 2012.

[51] A. Obata, E. Yoshimi, A. Waki et al., "Retention mechanism of hypoxia selective nuclear imaging/radiotherapeutic agent Cu-diacetyl-bis(N4-methylthiosemicarbazone) (Cu-ATSM) in tumor cells," Annals of Nuclear Medicine, vol. 15, no. 6, pp. 499-504, 2001.

[52] H. Kurihara, N. Honda, Y. Kono, and Y. Arai, "Radiolabelled agents for PET imaging of tumor hypoxia," Current Medicinal Chemistry, vol. 19, no. 20, pp. 3282-3289, 2012.

[53] Y. Minagawa, K. Shizukuishi, I. Koike et al., "Assessment of tumor hypoxia by 62Cu-ATSM PET/CT as a predictor of response in head and neck cancer: a pilot study," Annals of Nuclear Medicine, vol. 25, no. 5, pp. 1-7, 2011.

[54] D. W. Dietz, F. Dehdashti, P. W. Grigsby et al., "Tumor hypoxia detected by positron emission tomography with 60Cu-ATSM as a predictor of response and survival in patients undergoing neoadjuvant chemoradiotherapy for rectal carcinoma: a pilot study," Diseases of the Colon and Rectum, vol. 51, no. 11, pp. 1641-1648, 2008.

[55] F. Dehdashti, P. W. Grigsby, J. S. Lewis, R. Laforest, B. A. Siegel, and M. J. Welch, "Assessing tumor hypoxia in cervical cancer by PET with 60Cu- labeled diacetyl-bis(N4-methylthiosemicarbazone)," Journal of Nuclear Medicine, vol. 49, no. 2, pp. 201-205, 2008.

[56] F. Dehdashti, M. A. Mintun, J. S. Lewis et al., "In vivo assessment of tumor hypoxia in lung cancer with $60 \mathrm{Cu}$ ATSM," European Journal of Nuclear Medicine and Molecular Imaging, vol. 30, no. 6, pp. 844-850, 2003.

[57] J. P. Holland, J. S. Lewis, and F. Dehdashti, "Assessing tumor hypoxia by positron emission tomography with Cu-ATSM," Quarterly Journal of Nuclear Medicine and Molecular Imaging, vol. 53, no. 2, pp. 193-200, 2009.

[58] C. Catana, D. Procissi, Y. Wu et al., "Simultaneous in vivo positron emission tomography and magnetic resonance imaging," Proceedings of the National Academy of Sciences of the United States of America, vol. 105, no. 10, pp. 3705-3710, 2008.

[59] M. S. Judenhofer, H. F. Wehrl, D. F. Newport et al., "Simultaneous PET-MRI: a new approach for functional and morphological imaging," Nature Medicine, vol. 14, no. 4, pp. 459-465, 2008.

[60] T. E. Yankeelov, T. E. Peterson, R. G. Abramson et al., "Simultaneous PET-MRI in oncology: a solution looking for a problem?" Magnetic Resonance Imaging, vol. 30, no. 9, pp. 1342-1356, 2012.

[61] A. Boss, S. Bisdas, A. Kolb et al., "Hybrid PET/MRI of intracranial masses: initial experiences and comparison to PET/CT," Journal of Nuclear Medicine, vol. 51, no. 8, pp. 11981205, 2010.

[62] C. Buchbender, T. A. Heusner, T. C. Lauenstein, A. Bockisch, and G. Antoch, "Oncologic PET/MRI, part 1: tumors of the brain, head and neck, chest, abdomen, and pelvis," Journal of Nuclear Medicine, vol. 53, no. 6, pp. 928-938, 2012.
[63] C. Buchbender, T. A. Heusner, T. C. Lauenstein, A. Bockisch, and G. Antoch, "Oncologic PET/MRI, part 2: bone tumors, soft-tissue tumors, melanoma, and lymphoma," Journal of Nuclear Medicine, vol. 53, no. 8, pp. 1244-1252, 2012.

[64] S. Jbabdi, E. Mandonnet, H. Duffau et al., "Simulation of anisotropic growth of low-grade gliomas using diffusion tensor imaging," Magnetic Resonance in Medicine, vol. 54, no. 3, pp. 616-624, 2005.

[65] D. E. Woodward, J. Cook, P. Tracqui, G. C. Cruywagen, J. D. Murray, and E. C. Alvord, "A mathematical model of glioma growth: the effect of extent of surgical resection," Cell Proliferation, vol. 29, no. 6, pp. 269-288, 1996.

[66] P. K. Burgess, P. M. Kulesa, J. D. Murray, and E. C. Alvord Jr., "The interaction of growth rates and diffusion coefficients in a three-dimensional mathematical model of gliomas," Journal of Neuropathology and Experimental Neurology, vol. 56, no. 6, pp. 704-713, 1997.

[67] O. Clatz, M. Sermesant, P. Y. Bondiau et al., "Realistic simulation of the 3-D growth of brain tumors in MR images coupling diffusion with biomechanical deformation," IEEE Transactions on Medical Imaging, vol. 24, no. 10, pp. 13341346, 2005.

[68] P. Y. Bondiau, O. Clatz, M. Sermesant et al., "Biocomputing: numerical simulation of glioblastoma growth using diffusion tensor imaging," Physics in Medicine and Biology, vol. 53, no. 4, pp. 879-893, 2008.

[69] M. I. Miga, K. D. Paulsen, F. Kennedy, P. Hoopes, A. Hartov, and D. Roberts, "In vivo analysis of heterogeneous brain deformation computation for model updated image guidance," Computer Methods in Biomechanics and Biomedical Engineering, vol. 3, no. 2, pp. 129-146, 2000.

[70] C. H. Wang, J. K. Rockhill, M. Mrugala et al., "Prognostic significance of growth kinetics in newly diagnosed glioblastomas revealed by combining serial imaging with a novel biomathematical model," Cancer Research, vol. 69, no. 23, pp. 9133-9140, 2009.

[71] K. R. Swanson, H. L. P. Harpold, D. L. Peacock et al., "Velocity of radial expansion of contrast-enhancing gliomas and the effectiveness of radiotherapy in individual patients: a proof of principle," Clinical Oncology, vol. 20, no. 4, pp. 301-308, 2008.

[72] R. Fisher, "The wave of advance of advantageous genes," Annals of Eugenics, vol. 7, no. 4, pp. 355-369, 1937.

[73] R. Rockne, E. C. Alvord Jr., J. K. Rockhill, and K. R. Swanson, "A mathematical model for brain tumor response to radiation therapy," Journal of Mathematical Biology, vol. 58, no. 4-5, pp. 561-578, 2009.

[74] E. Hall, Radiobiology for the Radiologist, Lippincott, Philadelphia, Pa, USA, 1994.

[75] R. Rockne, J. K. Rockhill, M. Mrugala et al., "Predicting the efficacy of radiotherapy in individual glioblastoma patients in vivo: a mathematical modeling approach," Physics in Medicine and Biology, vol. 55, no. 12, pp. 3271-3285, 2010.

[76] N. C. Atuegwu, L. R. Arlinghaus, X. Li et al., "Integration of diffusion-weighted MRI data and a simple mathematical model to predict breast tumor cellularity during neoadjuvant chemotherapy," Magnetic Resonance in Medicine, vol. 66, no. 6, pp. 1689-1696, 2011.

[77] N. C. Atuegwu, D. C. Colvin, M. E. Loveless, L. Xu, J. C. Gore, and T. E. Yankeelov, "Incorporation of diffusion-weighted magnetic resonance imaging data into a simple mathematical model of tumor growth," Physics in Medicine and Biology, vol. 57, no. 1, pp. 225-240, 2012. 
[78] N. C. Atuegwu, J. C. Gore, and T. E. Yankeelov, "The integration of quantitative multi-modality imaging data into mathematical models of tumors," Physics in Medicine and Biology, vol. 55, no. 9, pp. 2429-2449, 2010.

[79] T. E. Yankeelov, N. C. Atuegwu, N. G. Deane, and J. C. Gore, "Modeling tumor growth and treatment response based on quantitative imaging data," Integrative Biology, vol. 2, no. 7-8, pp. 338-345, 2010.

[80] N. C. A. L. Atuegwu, X. Li, E. B. Welch, A. B. Chakravarthy, and T. E. Yankeelov, Parameterizing the Logistic Model of Tumor Growth by DW-MRI and DCE-MRI to Predict Breast Tumor Cellularity During Neoadjuvant Chemotherapy, Montreal, Canada, 2012.

[81] B. M. Ellingson, P. S. Laviolette, S. D. Rand et al., "Spatially quantifying microscopic tumor invasion and proliferation using a voxel-wise solution to a glioma growth model and serial diffusion MRI," Magnetic Resonance in Medicine, vol. 65, no. 4, pp. 1132-1144, 2011.

[82] B. M. Ellingson, T. F. Cloughesy, A. Lai, P. L. Nghiemphu, and W. B. Pope, "Cell invasion, motility, and proliferation level estimate (CIMPLE) maps derived from serial diffusion MR images in recurrent glioblastoma treated with bevacizumab," Journal of Neuro-Oncology, vol. 105, no. 1, pp. 91-101, 2011.

[83] B. Titz and R. Jeraj, "An imaging-based tumour growth and treatment response model: investigating the effect of tumour oxygenation on radiation therapy response," Physics in Medicine and Biology, vol. 53, no. 17, pp. 4471-4488, 2008.

[84] Y. Fujibayashi, H. Taniuchi, Y. Yonekura, H. Ohtani, J. Konishi, and A. Yokoyama, "Copper-62-ATSM: a new hypoxia imaging agent with high membrane permeability and low redox potential," Journal of Nuclear Medicine, vol. 38, no. 7, pp. 1155-1160, 1997.

[85] M. D. Szeto, G. Chakraborty, J. Hadley et al., "Quantitative metrics of net proliferation and invasion link biological aggressiveness assessed by MRI with hypoxia assessed by FMISO-PET in newly diagnosed glioblastomas," Cancer Research, vol. 69, no. 10, pp. 4502-4509, 2009.

[86] S. Gu, G. Chakraborty, K. Champley et al., "Applying a patient-specific bio-mathematical model of glioma growth to develop virtual $\left[{ }^{18} \mathrm{~F}\right]$-FMISO-PET images," Mathematical Medicine and Biology, vol. 29, no. 1, pp. 31-48, 2012.

[87] C. Vangestel, C. van de Wiele, N. van Damme et al., " ${ }^{99 m} \mathrm{Tc}$ $(\mathrm{CO})_{3}$ His-annexin A5 micro-SPECT demonstrates increased cell death by irinotecan during the vascular normalization window caused by bevacizumab," Journal of Nuclear Medicine, vol. 52, no. 11, pp. 1786-1794, 2011. 


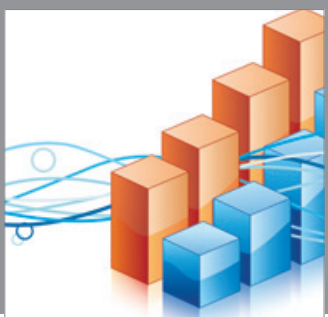

Advances in

Operations Research

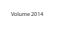

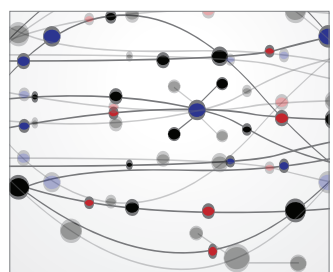

\section{The Scientific} World Journal
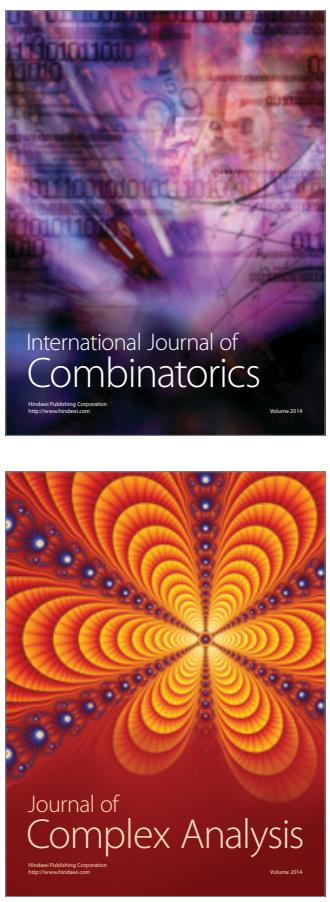

International Journal of

Mathematics and

Mathematical

Sciences
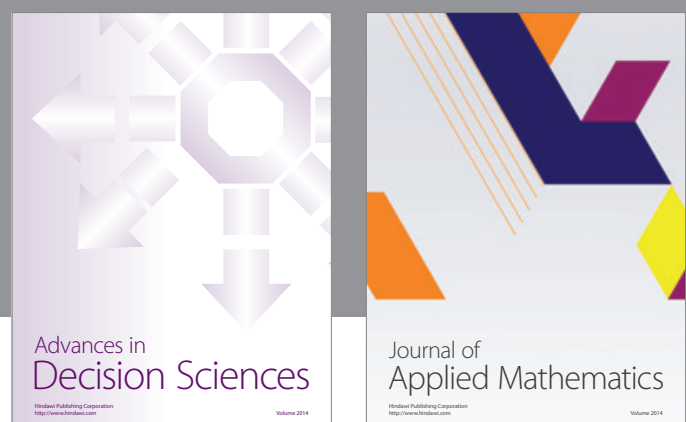

Journal of

Applied Mathematics
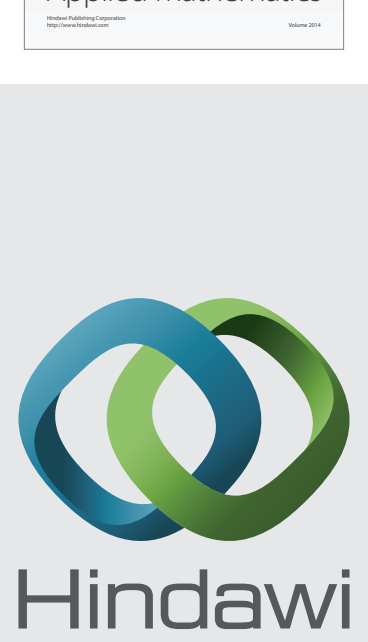

Submit your manuscripts at http://www.hindawi.com
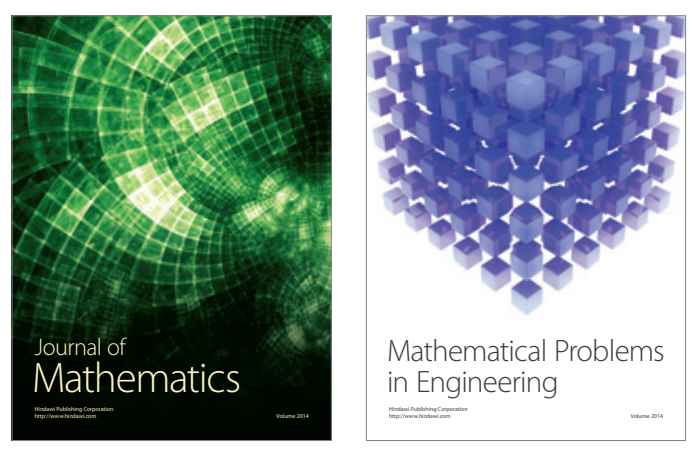

Mathematical Problems in Engineering
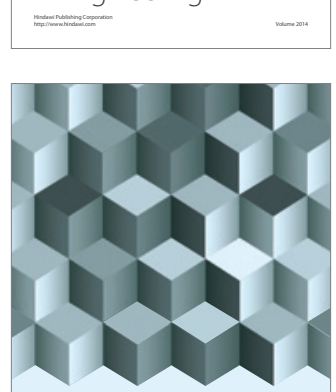

Journal of

Function Spaces
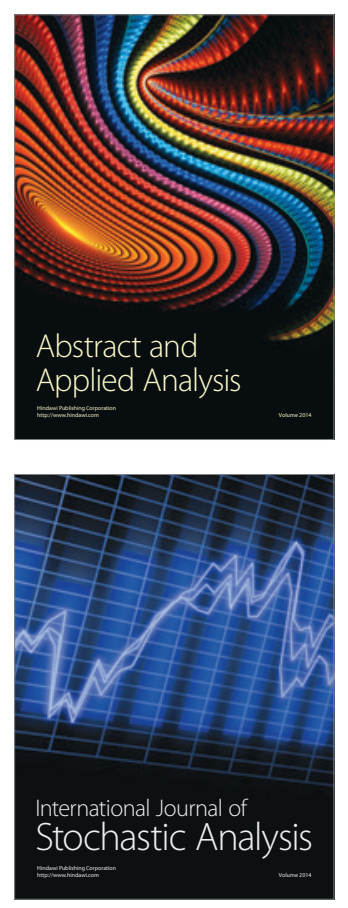

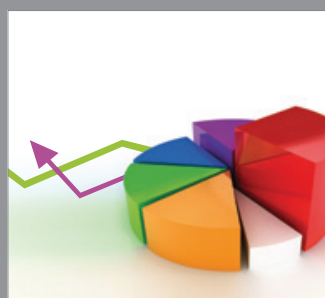

ournal of

Probability and Statistics

Promensencen
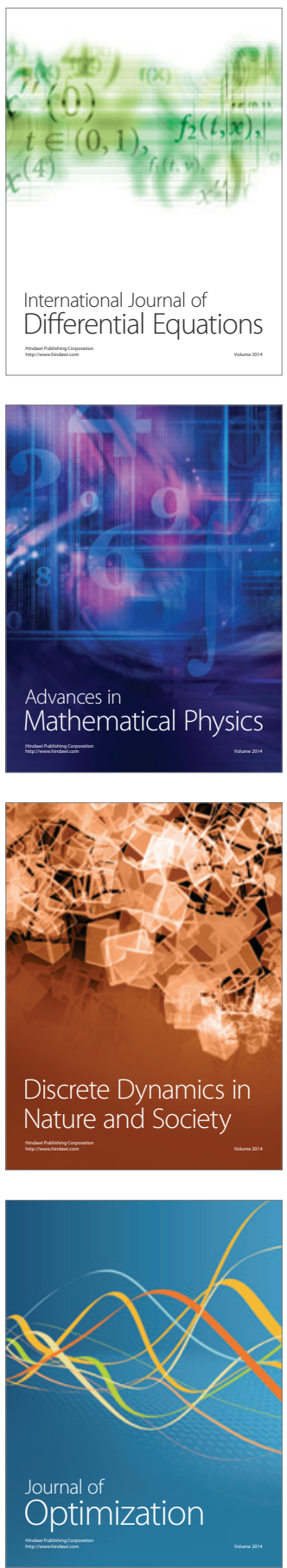